\title{
Impacts of obesity, maternal obesity and nicotinamide mononucleotide supplementation on sperm quality in mice
}

\author{
Neil A Youngson¹, G Mezbah Uddin ${ }^{1}$, Abhirup Das ${ }^{1,2}$, Carl Martinez ${ }^{1}$, Haley S Connaughton ${ }^{3}$, \\ Sara Whiting ${ }^{3}$, Josephine Yu ${ }^{1}$, David A Sinclair ${ }^{1,2}$, R John Aitken ${ }^{3}$ and Margaret J Morris ${ }^{1}$ \\ ${ }^{1}$ Department of Pharmacology, School of Medical Sciences, University of New South Wales, Sydney, New South \\ Wales, Australia, ${ }^{2}$ Paul F. Glenn Center for the Biological Mechanisms of Aging, Department of Genetics, Blavatnik \\ Institute, Harvard Medical School, Boston, Massachusetts, USA and ${ }^{3}$ School of Environmental and Life Sciences, \\ University of Newcastle, Callaghan, New South Wales, Australia
}

Correspondence should be addressed to M J Morris; Email: m.morris@unsw.edu.au

\begin{abstract}
Male fertility and sperm quality are negatively impacted by obesity. Furthermore, recent evidence has shown that male offspring from obese rat mothers also have reduced sperm quality and fertility. Here, we extend work in this area by comparing the effects of both maternal obesity and offspring post-weaning diet-induced obesity, as well as their combination, on sperm quality in mice. We additionally tested whether administration of the $\mathrm{NAD}^{+}$-booster nicotinamide mononucleotide (NMN) can ameliorate the negative effects of obesity and maternal obesity on sperm quality. We previously showed that intraperitoneal (i.p.) injection of NMN can reduce the metabolic deficits induced by maternal obesity or post-weaning dietary obesity in mice. In this study, female mice were fed a high-fat diet (HFD) for 6 weeks until they were $18 \%$ heavier than a control diet group. Thereafter, HFD and control female mice were mated with control diet males, and male offspring were weaned into groups receiving control or HFD. At 30 weeks of age, mice received $500 \mathrm{mg} / \mathrm{kg}$ body weight NMN or vehicle PBS i.p. for 21 days. As expected, adiposity was increased by both maternal and post-weaning HFD but reduced by NMN supplementation. Post-weaning HFD reduced sperm count and motility, while maternal HFD increased offspring sperm DNA fragmentation and levels of aberrant sperm chromatin. There was no evidence that the combination of post-weaning and maternal HFD exacerbated the impacts in sperm quality suggesting that they impact spermatogenesis through different mechanisms. Surprisingly NMN reduced sperm count, vitality and increased sperm oxidative DNA damage, which was associated with increased NAD ${ }^{+}$in testes. A subsequent experiment using oral NMN at $400 \mathrm{mg} / \mathrm{kg}$ body weight was not associated with reduced sperm viability, oxidative stress, mitochondrial dysfunction or increased $\mathrm{NAD}^{+}$in testes, suggesting that the negative impacts on sperm could be dependent on dose or mode of administration.

Reproduction (2019) 158 169-179
\end{abstract}

\section{Introduction}

Obesity can severely impact fertility and reproductive organ function (Cardozo et al. 2011, Liu \& Ding 2017). Human studies have shown that BMI inversely correlates with sperm count and various measurements of sperm quality including sperm motility, morphology and DNA damage (Jensen et al. 2004, Chavarro et al. 2010). Obesity reduces sperm quality through various means. These include changes in hormone levels such as leptin, testosterone and oestrogen (Considine et al. 1996, Chavarro et al. 2010) and an increase in the localised and systemic levels of oxidative stress (Gavriliouk \& Aitken 2015, Liu \& Ding 2017). Excess fat deposits have been shown to increase scrotal temperature in humans, which is thought to reduce sperm quality by denaturing enzymes that are required for spermatogenesis (Hjollund et al. 2000, Kort et al. 2006). In both humans and mice, obesity promotes the aromatisation of testosterone to estradiol (E2), which is known to impair spermatogenesis by reducing the level of testosterone in the body (Hjollund et al. 2000, Erdemir et al. 2012). In addition to $\mathrm{E} 2$, higher $\mathrm{BMI}$ was also found to reduce the levels of reproductive hormones such as inhibin B, follicle-stimulating hormone and luteinising hormone (Chavarro et al. 2010), which all play essential roles in spermatogenesis. Evidence from mice has also shown that obesity disrupts the blood-testis barrier, which may expose both the spermatogenic cells to bloodborne noxious agents and the seminiferous epithelium to autoimmune events (Fan et al. 2015). The correlation between sperm DNA damage and increased BMI (Kort et al. 2006, Chavarro et al. 2010, Dupont et al. 2013, Gavriliouk \& Aitken 2015) is thought to be linked to increased oxidative stress. While spermatozoa contain antioxidant enzymes, increased reactive oxygen species 
(ROS) levels in obesity are known to cause DNA damage and initiate apoptosis among spermatozoa, thereby decreasing sperm concentrations and increasing the likelihood of male infertility or failed pregnancies (Moustafa et al. 2004, Agarwal et al. 2014, Gavriliouk \& Aitken 2015).

A feature of the worldwide increase in obesity rates (World Health Organisation 2015) is the corresponding increase in maternal obesity, that is in women prior to and during pregnancy and lactation (Callaway et al. 2006, Reynolds et al. 2013). Maternal obesity is linked to increased offspring birth weight, adiposity (Martin et al. 2015, Starling et al. 2015) and a higher risk of pregnancy complications (Callaway et al. 2006, Avci et al. 2015, Sullivan et al. 2015). Postnatally, offspring from obese mothers have reduced survival rate (Reynolds etal. 2013), which is linked to increased risk for diseases such as CVD (Reynolds et al. 2013), metabolic syndrome (Boney et al. 2005) and non-alcoholic fatty liver disease (Oben et al. 2010). Maternal obesity can also reduce fertility in both male (Rodriguez-Gonzalez et al. 2015, Santos et al. 2015) and female (Ambrosetti et al. 2016, Tsoulis et al. 2016) offspring. Studies in rat have found oxidative stress levels to be elevated in the testis and sperm of offspring of obese mothers (Rodriguez-Gonzalez et al. 2015, Santos et al. 2015). Therefore, maternal obesity has the potential to not only negatively impact offspring health, but the health of grand-offspring via alteration of offspring sperm DNA or epigenetic state (Ge et al. 2014, Rodriguez-Gonzalez et al. 2015, Santos et al. 2015, Soubry et al. 2015). An additional consequence of maternal obesity is the exacerbation of negative health impacts as the offspring themselves are at a higher risk of becoming obese (Bahari et al. 2013, Uddin et al. 2017). This effect is driven by developmental programming of offspring metabolism and can lead to a more severe phenotype than 'first-generation obesity' (Starling et al. 2015, Elshenawy \& Simmons 2016).

$N A D^{+}$is a key metabolite, which also acts as a central signalling molecule and enzyme substrate with numerous roles in fundamental biological processes including energy metabolism, lifespan regulation, DNA repair, apoptosis and telomere maintenance (Yang \& Sauve 2016). Boosting $\mathrm{NAD}^{+}$levels through exercise (Uddin et al. 2016, 2017), supplementation with $\mathrm{NAD}^{+}$boosters, (usually precursor molecules) (Yoshino et al. 2011, Gariani et al. 2016, Uddin et al. 2016, Das et al. 2018) or increasing the activity of $\mathrm{NAD}^{+}$-dependent effectors (e.g. Sirtuin proteins) (Baur et al. 2006) have all been used effectively to recapitulate exercise-like benefits in obese and aged rodent models. We previously examined the effects of administration of the $\mathrm{NAD}^{+}$-booster nicotinamide mononucleotide (NMN) in maternal obesity by supplementing female offspring. We found improvements in offspring adiposity, metabolism (Uddin et al. 2017) and the reversal of some changes in markers of oocyte-secreted factor signalling (Bertoldo et al. 2018).
The effects of maternal obesity on offspring sperm quality are poorly understood. Considering the impact of the worldwide obesity epidemic on disease risk as well as fertility, there is a need to investigate this area more. Here, we add to the knowledge on how maternal obesity affects sperm quality in mice, as well as provide the first information on how NMN supplementation affects sperm quality in the context of normal weight, as well as obesity associated with both post-weaning and maternal HFD consumption. We hypothesised that similar to improvements in body-wide and liver metabolism, sperm quality would benefit from NMN supplementation in obese mice. However, our results suggest that in some circumstances NMN supplementation may actually be detrimental to sperm quality.

\section{Materials and methods}

\section{Animal procedures}

The effects of NMN on sperm was analysed in two mouse cohorts. One cohort investigated the effects of daily intraperitoneal (i.p.) injections of $\mathrm{NMN}$ on sperm from post-weaning HFD-induced obese mice and mice from a mother fed either a chow diet or a HFD. The second cohort investigated the effects of NMN administered in drinking water on sperm from chow-fed animals only, with experiments on live sperm focussing on measuring oxidative stress and mitochondrial function.

\section{Maternal obesity cohort design}

Female C57BL/6 mice (Animal Resource Centre, Canning Vale, WA, Australia) were housed in the Biological Resource Centre at $21^{\circ} \mathrm{C} \pm 2$, with a $12 \mathrm{~h} / 12 \mathrm{~h}$ light cycle. Mice were housed four per cage and fed standard chow pellets $(11 \mathrm{~kJ} / \mathrm{g}$, $4 \%$ total fat, 13\% digestible energy from lipids, Gordon's Stock Feeds, Yanderra, NSW, Australia). At 4 weeks of age, females were separated into high-fat and control chow diets. Highfat diet (HFD) was $19 \mathrm{~kJ} / \mathrm{g}$, composed of $23.5 \%$ total fat, $43 \%$ digestible energy from lipids (Specialty Feeds, Glen Forrest, WA, Australia). When HFD-fed females weighed 18\% more than control mice (between 9 and 10 weeks), they were mated with males (same provider) fed with standard show diet (four females and one male per cage). To control for parity effects only virgin females were bred. Due to the expected low pregnancy rates and high levels of cannibalism in first-litters (especially in HFD-fed females), and the use of inexperienced virgin males, we started with 48 chow-fed and 80 HFD-fed females. Pregnancy was confirmed by body weight gain 1 week post mating, after which dams were housed one per cage and maintained on their pre-pregnancy diet throughout pregnancy and lactation. Dams gave birth at approximately 12-13 weeks of age. All pups were born over a 3-day period and were left undisturbed for the first week to prevent maternal stress. At PND 24, each mouse received an ear punch. For all molecular analyses, the use of this individual identifier ensured that experimenters were blind to treatment group of the animals. 
At PND 28 male offspring were weaned and distributed across chow or HFD groups, ensuring that the group average body weights were as near to equivalent as possible (with the only differences being due to the dam's diet, that is all group average weights were larger if the dam was fed HFD). Siblings were split between groups as often as possible to control for litter-effects which resulted in the 10-12 pups in each group being from a minimum of six dams.

Pups were weaned onto either HFD or control diet; diets were maintained through to 34 weeks of age. A subgroup of offspring from both maternal diet groups was given daily i.p. injections of $\mathrm{NMN}$ (500 mg/kg body weight) from 31 to 34 weeks of age. All non-NMN-treated offspring received daily injection of PBS vehicle. Each group containing 10-12 offspring were killed at 34 weeks. The experimental timeline and groups are illustrated in Fig. $1 \mathrm{~A}$ and $\mathrm{B}$, respectively. Tissues of interest were harvested at sacrifice and snap-frozen in liquid nitrogen and stored at $-80^{\circ} \mathrm{C}$. All animal procedures were approved by Animal Ethics Committee, UNSW (Ethics number 13/25B).

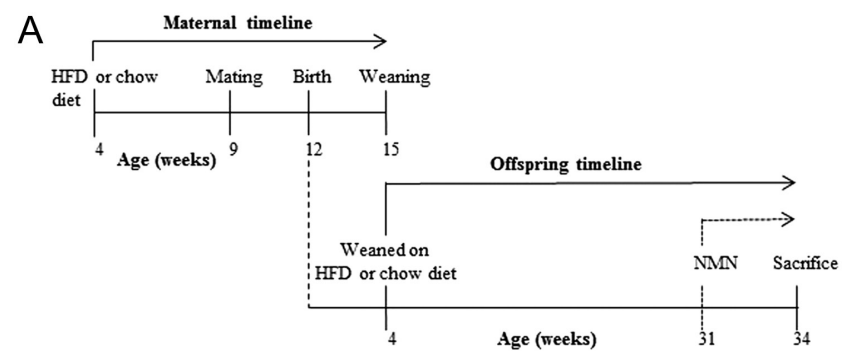

B

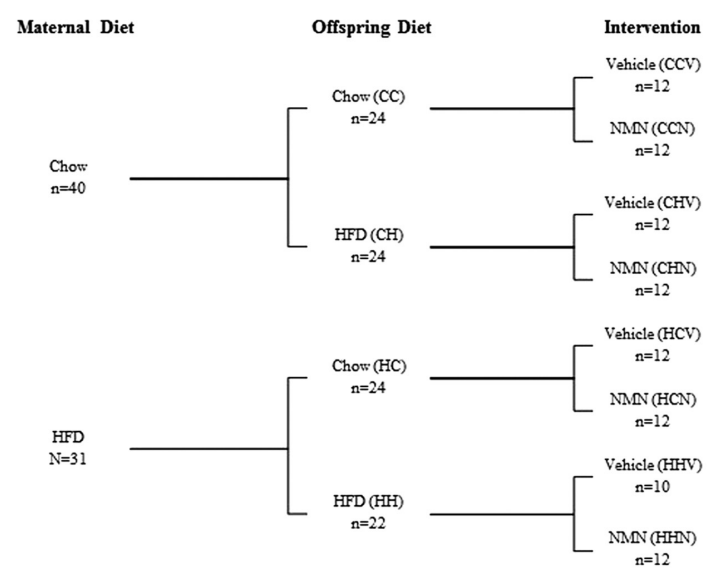

Figure 1 Offspring timeline and maternal obesity cohort design. (A) Male offspring of mothers were weaned on HFD or chow diet at 4 weeks of age until cull (33 weeks). At 31 weeks of age, a subgroup of offspring from both maternal diet groups was injected (i.p.) with NMN daily. (B) Mothers were fed with either chow diet (C) or HFD (H). Offspring of both maternal groups were fed with either chow diet (CC and $\mathrm{HC}$ ) or HFD (CH and $\mathrm{HH}$ ). Nicotinamide mononucleotide $(\mathrm{NMN})$ was injected, intraperitoneally, in four groups $(\mathrm{CCN}, \mathrm{CHN}$, $\mathrm{HCN}$ and $\mathrm{HHN}$ ). Phosphate buffered saline vehicle was injected in the other four groups (CCV, CHV, HCV, HHV).

\section{Oral administration of NMN cohort design}

Two-month-old male C57BL/6 mice were housed in the Biological Resource Centre at UNSW and fed the standard chow diet. Half of the cohort was given NMN $(400 \mathrm{mg} / \mathrm{kg}$ body weight) via drinking water for 24 weeks; controls received drinking water. At 6 months, mice were killed and tissue was collected. All animal procedures were approved by Animal Ethics Committee, UNSW (ACEC 16/13B).

\section{Sperm collection}

Sperm was collected from vas deferentia and cauda epididymides of mice into $1 \mathrm{~mL}$ of DMEM or, if FACS was to be performed in $1 \mathrm{~mL}$ of Biggers, Whitten and Whittingham media (BWW). Motility, vitality and concentration were assessed under a light microscope using a haemocytometer. To examine sperm motility, $5 \mu \mathrm{L}$ sperm suspension were added to $45 \mu \mathrm{L}$ BWW working solution. For sperm count, $5 \mu \mathrm{L}$ sperm suspension were added to $45 \mu \mathrm{L}$ sperm diluting fluid $\left(2.5 \mathrm{~g} \mathrm{NaHCO}_{3}, 0.5 \mathrm{~mL}\right.$ formaldehyde and $49.5 \mathrm{~mL}$ in water). For vitality $5 \mu \mathrm{L}$ sperm suspension were added to $5 \mu \mathrm{L}$ eosin stain $(0.5 \% \mathrm{w} / \mathrm{v}$ eosin, $0.9 \% \mathrm{w} / \mathrm{v} \mathrm{NaCl}$ in water $)$ and immediately examined by haemocytometer. Sperm with pink heads were considered to be non-viable. Concurrent with the vitality measurement, sperm motility was assessed by counting the percentage of sperm that were moving (either slowly or vigorously). For FACS assays viability was measured with 7-Aminoactinomycin D (7-AAD). Aliquots containing a million sperm (in approximately $200 \mu \mathrm{L}$ DMEM) were snap-frozen in liquid nitrogen and stored for 8-hydroxy-2'deoxyguanosine (8-OHdG), halo, chromomycin A3 (CMA3) and sperm chromatin structure assay (SCSA) assays. For FACS experiments aliquots of the sperm suspension were further diluted in BWW to make multiple $190 \mu \mathrm{L}$ aliquots of $10^{6} / \mathrm{mL}$ sperm for staining.

\section{Frozen sperm assays}

Oxidative DNA damage (8-OHdG) immunofluorescence (Houston et al. 2018) and CMA3 staining (Smith et al. 2013) were performed with published laboratory protocols. For 8-OHdG measurement, spermatozoa were snap-frozen in liquid nitrogen and stored at $-80^{\circ} \mathrm{C}$ until analyses could occur. These cells were then resuspended in primary DNA/ RNA damage antibody (Novus Biologicals) $(25 \mu \mathrm{g} / \mathrm{mL}$ in PBST) overnight at $4^{\circ} \mathrm{C}$. After primary incubation, spermatozoa were washed in PBS and then incubated with Alexa Fluor 488 goat $\alpha$ mouse secondary $\left(5 \mu \mathrm{g} / \mathrm{mL}\right.$ in PBST) for $1 \mathrm{~h}$ at $37^{\circ} \mathrm{C}$. Cells were washed twice in PBS and viewed by fluorescence microscopy. A sample of 100 cells was assessed, scoring positive by the presence of nuclear fluorescence. 8-OHdG was chosen for as a marker for sperm oxidation as it has been used as such in human (Vorilhon et al. 2018) and rodent (Kocer et al. 2015) studies of environmentally induced (Ahmed-Farid et al. 2017) and genetically induced (Huang et al. 2016) sperm DNA damage.

For CMA3 measurement spermatozoa were frozen in liquid nitrogen and thawed at room temperature. Cells were centrifuged and resuspended cells into Mcllvaine buffer 
$(17 \mathrm{~mL}$ of $0.1 \mathrm{~mol} / \mathrm{L}$ citric acid mixed with $83 \mathrm{~mL}$ of $0.2 \mathrm{~mol} / \mathrm{L}$ $\mathrm{Na}_{2} \mathrm{HPO}_{4}$ and $10 \mathrm{mmol} / \mathrm{L} \mathrm{MgCl}$, $\mathrm{pH}$ 7.0). The chromatin of sperm cells were labelled by incubating with $25 \mu \mathrm{L}$ of CMA3 solution $(0.25 \mathrm{mg} / \mathrm{mL}$ in Mcllvaine buffer) for $20 \mathrm{~min}$ in the dark at room temperature. Cells were washed with Mcllvaine buffer before counting labelled cells under fluorescent microscope.

The Sperm Chromatin Dispersion Test (halo) assay was a modification of a previously published protocol (Fernandez et al. 2003). Briefly, spermatozoa were frozen in liquid nitrogen and thawed at room temperature. Cells were mixed with $1 \%$ low melting agarose to a final concentration of agarose $0.7 \%$, at $37^{\circ} \mathrm{C}$. The cell-agarose mixture was put on to a slide precoated with $0.65 \%$ standard agarose. Slides were solidified at $4^{\circ} \mathrm{C}$ for $4 \mathrm{~min}$. Coverslip was carefully removed and placed horizontally in acid denaturation solution $(0.08 \mathrm{~N} \mathrm{HCl})$ for $7 \mathrm{~min}$ at room temperature. Slides are then incubated in a neutralising and lysing solution 1 (0.4M Tris, 0.8M DTT, 1\% SDS and $50 \mathrm{mM}$ EDTA, pH 7) for $10 \mathrm{~min}$ at room temperature, then into neutralising and lysing solution 2 (0.4M Tris, $2 \mathrm{M}$ $\mathrm{NaCl}$ and $1 \%$ SDS, $\mathrm{pH} 7.5$ ) for $5 \mathrm{~min}$ at room temperature. Slides are then washed in Tris-borate-EDTA buffer (0.09 M Trisborate and $0.002 \mathrm{M}$ EDTA, $\mathrm{pH} 7.5$ ) for $2 \mathrm{~min}$, dehydrated in 70,90 and $100 \%$ ethanol for 2 min each at room temperature and air dried. Slides were stained with DAPI for $10 \mathrm{~min}$ at room temperature. Slides were counted under a fluorescent microscope for 'halo' or 'no halo'.

The SCSA performed as described previously with modification (Evenson \& Jost 2000). Briefly, spermatozoa were snap-frozen in liquid nitrogen and stored at $-80^{\circ} \mathrm{C}$ until further analysis. The spermatozoa were thawed and placed on ice. Hundred microlitres of the sperm suspension was added to a FACS tube with $200 \mu \mathrm{L}$ of acid detergent solution. After exactly $30 \mathrm{~s}$, spermatozoa were stained with $600 \mu \mathrm{L}$ of acridine orange staining solution. Using a FACScanTM Flow Cytometer (BD) debris was gated out and 5000 sperm events were acquired per sample. The ratio of single stranded (red) to double stranded (green) fluorescence (\%DFI) was calculated using CellQuestTM software (BD).

\section{Mitochondrial DNA (mtDNA) copy number measurement}

This was performed by qPCR on isolated sperm DNA with primers for mtDNA in the Cytb gene with normalisation to the nuclear genome in the Rplpo gene as previously described (Uddin et al. 2016).

\section{Live sperm FACS staining}

Samples from each mouse were assessed for viability, mitochondrial membrane potential (MMP, $\Psi M \%$ ), and oxidative stress using the following dyes: 7-Aminoactinomycin D (7-AAD) (BD Biosciences), JC-1 dye (Life Technologies) and MitoSOX Red (MSR) Mitochondrial Superoxide Indicator (Life Technologies). The component of each tube used to examine MMP and oxidative stress are as described in Supplementary Table 1 (see section on supplementary data given at the end of this article). All tubes were incubated at $37^{\circ} \mathrm{C}\left(5 \% \mathrm{CO}_{2}\right)$ for $15 \mathrm{~min}$ then centrifuged for $3 \mathrm{~min}$ at room temperature, $1500 \mathrm{~g}$.
The supernatant was aspirated and pellets were redissolved in $400 \mu \mathrm{L}$ of BWW working solution. Samples were kept on ice before evaluation by flow cytometry 10 min later. The time from removal of cauda epididymis from the mouse until initiation of FACS analysis was approximately $30 \mathrm{~min}$.

\section{Flow cytometry}

Flow cytometry was conducted at the Biological Resource Imaging Laboratory (BRIL) of the Mark Wainwright Analytical Centre (UNSW, Australia). Samples were evaluated using a BD FACSVerse $^{\mathrm{TM}}$ flow cytometer (BD Biosciences, CA, USA) and acquisitions were obtained using the BD FACSuite ${ }^{T M}$ software (BD Biosciences). Dyes were excited by a $488 \mathrm{~nm}$ laser. Overall, 50,000 events were acquired for each sample. Data were analysed using the Flowjo v10.2 software (FlowJO LLC, Oregon, USA). A forward-scatter and side-scatter cytogram were used to identify and gate for sperm cells as described in a previous study (Yeung et al. 2002) (Supplementary Fig. 1A). From this gated population, viable sperm cells were identified as 7-AAD negative (7-AAD -ve; emission wavelength $647 \mathrm{~nm}$ ) and gated for subsequent JC-1 or MSR analyses (Supplementary Fig. $1 \mathrm{~B}$ and $\mathrm{C}$ ). Results were expressed as the percentage of 7-AAD -ve sperm cells. Only gated viable sperm were examined for oxidative stress or MMP.

To examine oxidative stress levels, viable sperm cells were divided into two groups: those with low MSR fluorescence and with high MSR fluorescence, as detected with a phycoeryhtrin (PE) filter (emission wavelength $578 \mathrm{~nm}$ ) which are representative of low and high oxidation, respectively (Supplementary Fig. 1D and E). Results were expressed as the percentage of live sperm cells positive for high MSR.

To examine MMP, the viable sperm cells were plotted on a fluorescein isothiocyanate (FITC; emission wavelength $519 \mathrm{~nm}$ ) versus phycoerythrin (PE) cytogram (Supplementary Fig. 1F). FITC and PE measures the green and orange fluorescence emitted by JC-1, respectively. The cytogram was divided into quadrants (Supplementary Fig. 1F) as described by Binet et al. (2014). Results are expressed as the percentage of live sperm in the high orange (HO) Q1 or high green (HG) Q3 or the ratio between $\mathrm{HO}$ and $\mathrm{HG}$ fluorescence that is the ratio between Q1+Q2 and Q2+Q3, respectively.

\section{Measurement of testes $\mathrm{NAD}^{+}$and NADH levels}

Testes were homogenised in extraction buffer $(50 \mathrm{mM}$ Tris $\mathrm{HCl}$, $10 \mathrm{mM}$ nicotinamide, $0.1 \%$ Triton $\mathrm{X}-100)$ and centrifuged ( $7000 \mathrm{~g}$ for $5 \mathrm{~min}$ at $4{ }^{\circ} \mathrm{C}$ ). An aliquot of the supernatant was taken for protein quantification. The remainder of the supernatant was passed through $\left(14,000 \mathrm{~g}\right.$ for $45 \mathrm{~min}$ at $\left.4^{\circ} \mathrm{C}\right)$ a $10 \mathrm{kDa}$ filter (Amicon) and flowthrough was spilt into two aliquots. One was placed on ice to measure total NAD while the other was used to determine $\mathrm{NAD}^{+}$content through acidification and heat treatment before being placed back on ice. Samples were then reacted with alcohol dehydrogenase on a 96-well plate at room temperature, followed by quantification using the BioRad iMark microplate reader. All steps apart from the filtering of lysate through a column were performed as previously described (Uddin et al. 2016). 


\section{Statistical analyses}

Maternal obesity cohort: GraphPad Prism v6.0 software (GraphPad Software) was used to assess the normality of the data using the D'Agostino-Pearson omnibus test. Statistical analyses were performed using IBM SPSS Statistics 22 (IBM Corp.). Three-way ANOVA was performed to examine the effects of maternal diet, offspring diet and intervention on phenotype measurements. Simple main effects were performed to examine any significant effect caused by an interaction between these factors. Post-hoc comparisons were Bonferroni corrected. Results are expressed as mean \pm S.E.M. Results were considered significant if $P \leq 0.05$.

Oral administration cohort: Two-way ANOVA was performed to analyse the effects of NMN on oxidative stress and MMP. Student's $t$-test was used to examine the effects of NMN versus control on live sperm cohort. Results were considered significant if $P \leq 0.05$.

\section{Results}

\section{Maternal obesity cohort}

\section{Effects on body weights and offspring testes}

Prior to mating, after 6 weeks on the HFD, dams were $18 \%$ heavier than control dams (17 chow dams average $17.4 \pm 1.0 \mathrm{~g}$, $14 \mathrm{HFD}$ dams average $20.7 \pm 1.3 \mathrm{~g}$, $P=7 \times 10^{-9}$ ) due to an increase in adiposity (Uddin et al. 2017). Both maternal diet and offspring postweaning HFD increased offspring body weight as expected (Fig. 2A). NMN supplementation reduced overall body weight (Fig. 2A). Adiposity, as determined by retroperitoneal fat pad mass at sacrifice followed the same trend, with increases due to post-weaning HFD (>500\%), due to maternal obesity (>200\%) and the combination of maternal obesity and post-weaning HFD (>700\%) compared to chow-fed mice from chowfed mothers, while NMN reduced adiposity in the HFD-fed mice (data in Uddin et al Manuscript Under Review). Both maternal and offspring post-weaning HFD increased testis weight (Fig. 2B). The maternal diet associated increase in testis weight was still significant when weight was divided by the length of the mouse; however, the post-weaning diet effect was lost (Supplementary Table 2).

\section{Effects on live sperm measurements}

Post-weaning HFD and NMN both reduced sperm count (Fig. 2C). Sperm motility was reduced by post-weaning HFD only (Fig. 2D). Sperm vitality was decreased by NMN treatment (where an eosin-stained head was considered to indicate a dead sperm) (Fig. 2E).

\section{Effects on frozen sperm quality}

NMN treatment had a striking effect on 8-OHdG levels in sperm, increasing it in all groups, compared to the corresponding control group (Fig. 2F). No significant
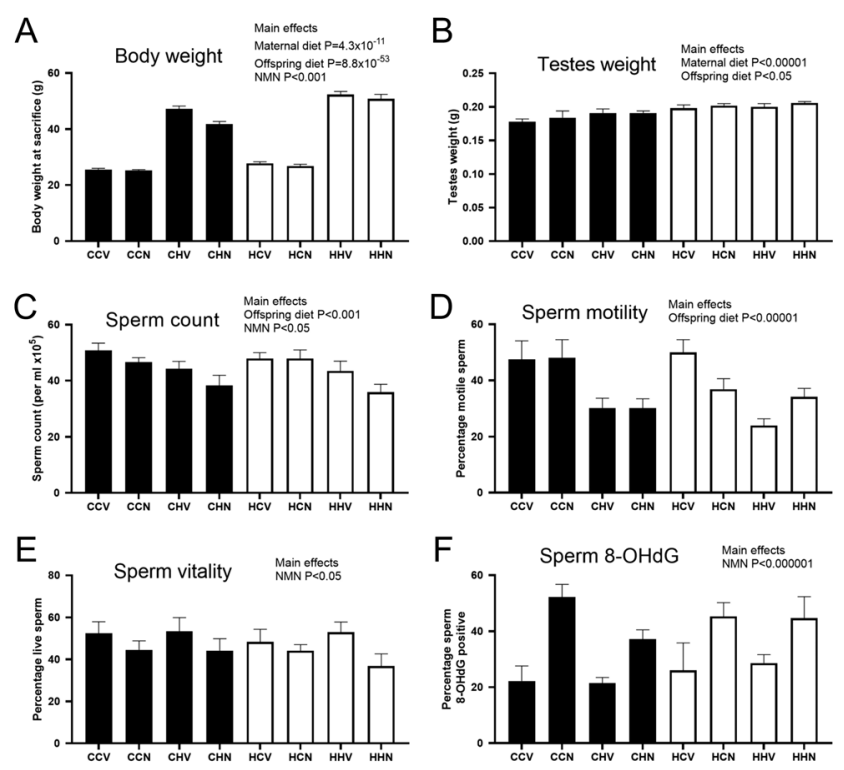

Figure 2 Effects of maternal obesity, offspring post-weaning diet and intraperitoneal NMN injection on anthropometric $(A, B)$ and sperm parameters in offspring $(\mathrm{C}-\mathrm{F})$ in offspring. The first letter of the group code indicates maternal diet, chow $(\mathrm{C})$ or HFD $(\mathrm{H})$. The second letter indicates offspring post-weaning diet, chow $(\mathrm{C})$ or HFD $(\mathrm{H})$. The third letter indicates NMN (N) or vehicle $(\mathrm{V})$ injection. Black bars indicate offspring from chow-fed mothers, white bars indicate offspring from HFD-fed mothers. $P$ values from a three-way ANOVA that was used to compare all eight groups where maternal diet, post-weaning diet and treatment were three different factors. Data are mean \pm S.E.M.

effects were seen in the halo assay and variation was large in this measurement. CMA3 staining was increased by maternal diet (Supplementary Table 2), although the percentage of sperm that stained were low in all groups. In the SCSA, the \%DFI was increased by maternal diet, and there was a trend for offspring HFD to increase it (Supplementary Table 2).

\section{mtDNA copy number}

Post-weaning HFD reduced mitochondrial copy number (Supplementary Table 2). The effects of NMN were more complex, as it increased copy number in offspring from chow-fed mothers but decreased it offspring from HFD mothers (Supplementary Table 2).

\section{Oral administration of NMN cohort}

To further our investigation into the effects of NMN administration on sperm quality, we undertook a second cohort. This aimed to uncover whether NMN affected sperm when the drug is orally administered. Mice were maintained on chow diet with access to vehicle (water) or $400 \mathrm{mg} / \mathrm{kg}$ NMN for 24 weeks. There was no difference in the body weights of the 32-week-old males (Supplementary Table 3). Sperm count, motility and mitochondrial copy number also were not significantly different between the two groups (Supplementary Table 3). 


\section{FACS analysis of viability, oxidative stress and MMP in oral administration of NMN cohort}

We examined live sperm with a FACS assay that measured incorporation of the dye 7-AAD as an indicator of viability. However, NMN had no significant effect on viability (Fig. 3A). To measure sperm oxidative stress, and MMP, we treated sperm with MitoSOX Red and JC-1 dyes, respectively. As positive controls, we treated sperm with arachidonic acid to increase sperm oxidative stress and FCCP to reduce MMP. Both arachidonic acid (AA) and carbonyl cyanide-trifluoromethoxyphenylhydrazone (FCCP) reduced viability in both tests $(P \leq 0.001)$ (Fig. 3A and $C$ ). Unexpectedly, the percentage of live sperm that is low 7-AAD staining was lower in the sperm used in the oxidative stress assay (that were stained with 7-AAD and MSR) than the sperm used to investigate MMP (stained with 7-AAD and JC-1) (Fig. 2A vs C), despite the experiments using aliquots of sperm from the same mice. This could be due to the MSR and JC-1 stains themselves having differential impacts on sperm viability or on the intensity of 7-AAD fluorescence. However, as the experimental
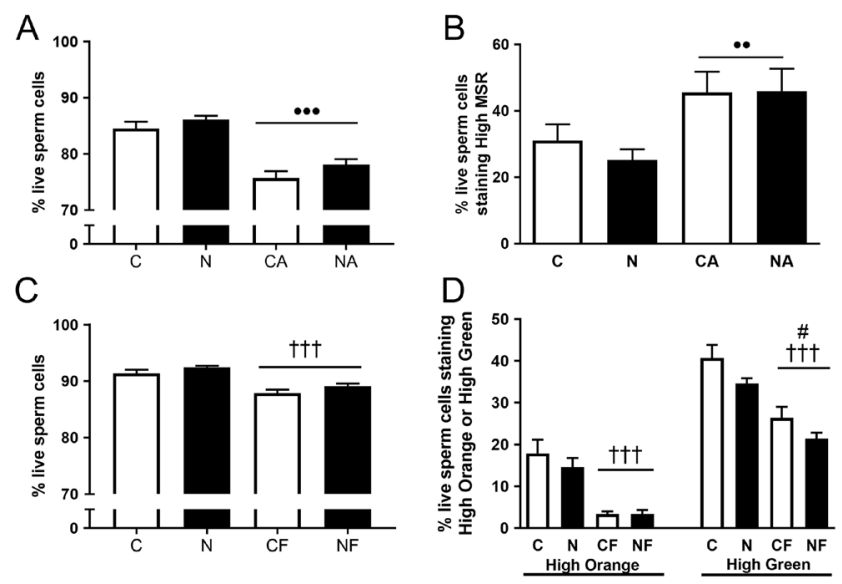

Figure 3 Viability, oxidative stress and mitochondrial membrane potential of sperm from control and NMN-treated mice in the Oral Administration Cohort. (A) Viability as determined by 7-AAD staining of sperm used for oxidative stress measurement. $\bullet P \leq 0.01$ reduction in viability due to positive control AA. (B) Oxidative stress as determined by mitosox red (MSR) staining. $\cdots P \leq 0.001$ increase in oxidative stress due to positive control arachidonic acid (AA). (C) Viability as determined by 7-AAD staining of sperm used for mitochondrial membrane potential measurement. ${ }^{t+t} P \leq 0.001$ reduction in viability due to positive control FCCP. (D) Mitochondrial membrane potential as determined by JC- 1 staining. ${ }^{+t+} P \leq 0.001$ reduction in $\mathrm{HO}$ and high green-staining sperm due to positive control FCCP. ${ }^{\sharp} P \leq 0.05$ reduction in high green-staining sperm due to NMN. C, Control mouse sperm; N, NMN mouse sperm; CA, control mouse sperm treated with $\mathrm{AA}$; NA, NMN mouse sperm treated with AA; CF, control mouse sperm treated with FCCP; NF, NMN mouse sperm treated with FCCP. Data are shown as mean \pm S.E.M., $n=10-13$ per group. Black bars are NMN-treated groups. Data were analysed using two-way ANOVA. Note Fig. 3A and B are measurements from the same sperm aliquot. Figure $3 \mathrm{C}$ and $\mathrm{D}$ are measurements of a different sperm aliquot, but from the same mice as for Fig. 3A and B. Data are mean \pm S.E.M. groups were balanced these unexpected effects did not obstruct the investigation of the effects of NMN.

As expected AA significantly increased high MSR levels $(P=0.003)$ (Fig. 3B). However, there was no significant effect of NMN on high MSR levels (Fig. 3B). Looking at MMP, two separate two-way ANOVAs were performed to look at the effect of NMN on HO levels and its effect on $\mathrm{HG}$ levels. JC-1 is predominantly a monomer at low MMP and yields green fluorescence. At high MMP, JC-1 aggregates and yields an orange emission. Therefore, the fluorescence reflects the amount of mitochondria that are in a state of high or low MMP. As expected of a mitochondrial uncoupler, FCCP significantly decreased sperm viability $(P \leq 0.001)$ (Fig. $3 C)$. Analysis revealed that there was no significant effect of $\mathrm{NMN}$ on $\mathrm{HO}$ levels. However, there was a significant reduction of $\mathrm{HG}$ level when treated with NMN $(P=0.037)$ (Fig. 3C).

\section{Measurement of testes $\mathrm{NAD}^{+}$and $\mathrm{NADH}$ levels}

$\mathrm{NAD}^{+}$levels were doubled in the testes of mice that received i.p. NMN injections compared to those receiving vehicle $\mathrm{PBS}$ (Fig. $4 \mathrm{~A}, P=5 \times 10^{-5}$ ). There was also a trend for $\mathrm{NADH}$ to be increased (Fig. 4B, $P=0.15$ ). However, no differences in $\mathrm{NAD}^{+}$or $\mathrm{NADH}$ were seen in the testes of mice receiving $\mathrm{NMN}$ in drinking water (Fig. 4C and D).

\section{Discussion}

Our maternal obesity cohort allowed examination of the effects of two periods of HFD exposure ('maternal' i.e. while in utero and during lactation, and post-weaning)
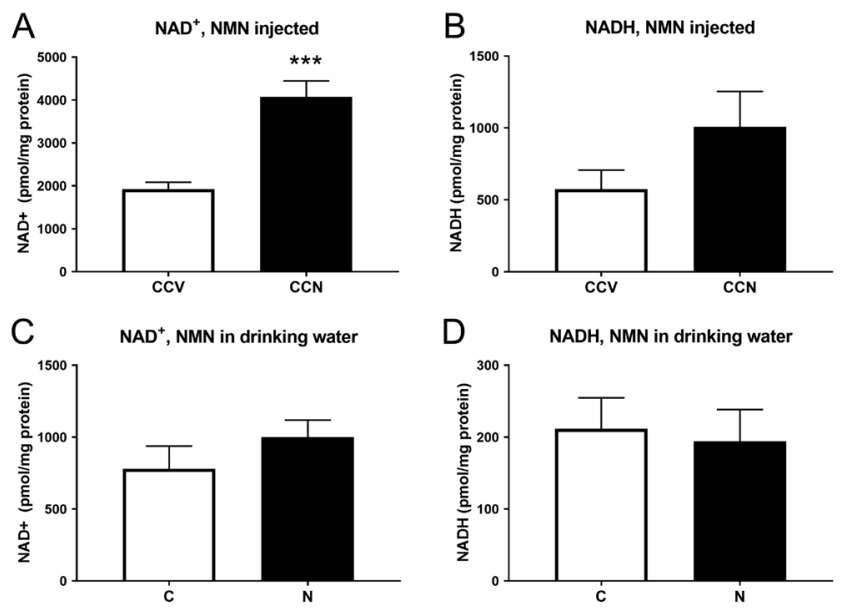

Figure $4 \mathrm{NAD}^{+}$and $\mathrm{NADH}$ levels in testes of mice from both cohorts (NMN injected and NMN in drinking water). Daily intraperitoneal injection of $500 \mathrm{mg} / \mathrm{kg}$ body weight NMN (A) increased $\mathrm{NAD}^{+}$levels in testes $\left({ }^{* * *} P=5 \times 10^{-5}\right)$ while (B) NADH levels had a trend for increase $(P=0.15)$. Daily consumption $400 \mathrm{mg} / \mathrm{kg}$ body weight NMN in drinking water did not increase (C) NAD ${ }^{+}$or (D) NADH in testes. Data are mean \pm S.E.M., $n=9 / 10$ per group in the i.p. cohort and 5/10 in the drinking water cohort. 
on widely used indicators of sperm quality. Additionally, effects of the $\mathrm{NAD}^{+}$boosting drug, NMN, on sperm quality were examined. As far as we are aware, no previous studies have compared the effects of dietinduced obesity, maternal obesity and their combination on sperm quality.

The effects on offspring body weights were profound with the expected increases observed due to both maternal HFD and post-weaning HFD. The combination of HFD during the two time periods exacerbated the effect on body weight, so that the mice with the greatest body weights were the $\mathrm{HH}$ groups (a doubling in body weight compared to the CC groups), as has also been observed in earlier studies (Bahari et al. 2013, Uddin et al. 2017). A reduction in sperm count and motility due to post-weaning HFD mirrors the situation observed in many human obesity studies (reviewed in Craig et al. 2017). Similarly, the minor effects of post-weaning HFD on sperm DNA and chromatin integrity in our experiment (only a trend in \%DFI, $P=0.06$ ) may also recapitulate the human situation, as some studies have found associations between high BMI and high DFI (Kort et al. 2006), but others have not (Bandel et al. 2015, Oliveira et al. 2018). The reduction in mtDNA copy number due to post-weaning HFD may be an indicator of sperm mitochondrial dysfunction (Malik \& Czajka 2013, Oliveira et al. 2018), although mouse experiments have shown that fertility can be maintained even with a genetically induced threefold reduction in mtDNA (Wai et al. 2010).

Few studies in humans and animals have examined the relationship between maternal obesity and male offspring sperm quality. Two studies from members of the same group (Rodriguez-Gonzalez et al. 2015, Santos et al. 2015) showed that male pups of obese rat dams had reduced fertility and poor sperm quality, based on sperm parameters (reduced concentration, \% viability and \% motility) compared to male pups of non-obese dams. They also observed several changes that highlighted increases in offspring sperm oxidative stress due to maternal obesity. Even though we detected increased CMA3 and SCSA DFI, indicating that maternal obesity impacted sperm chromatin (Supplementary Table 2), we did not observe changes to sperm concentration, viability and motility, nor an increase in the oxidative stress marker 8-OHdG. Additionally, the brothers of the mice used in this study were mated to enable an examination of the F2 generation (unpublished), but no changes in fertility (as determined by number of litters sired and average pups per litter) were seen (data not shown). These differences are likely to be due to speciesand diet-specific impacts of maternal obesity or possibly the extent of weight gain achieved in this study.

In the two rat studies by Santos et al. (2015) and Rodriguez-Gonzalez et al. (2015), the male pups of obese and control dams received only chow diet postweaning so only had high levels of adiposity when their rats were aged to nearly 2 years. Interestingly, in those studies, the impacts on sperm oxidative stress, concentration, viability and even fertility preceded the age-associated extreme adiposity suggesting that the negative effects of maternal obesity on male offspring reproduction may not be dependent on obesity. In support of this, in our study, it was interesting to note that while the body weight (Fig. 2A) and adiposity of the mice was exacerbated by the combination of maternaland post-weaning HFD, there were no obvious additive effects in any of the sperm parameters. This may suggest that the effects of maternal obesity occur primarily through alteration of early developmental processes, in prospermatogonia for example, while obesity-induced post-weaning affects the later stages of spermatogenesis (Christante et al. 2013).

The only human study to date on the effects of maternal obesity on offspring sperm had a relatively small sample size ( $n=25$ sons of mothers with pre-pregnant BMI $>25$ vs $n=265$ from mothers with BMI 18.5-24.99) (RamlauHansen et al. 2007). Trends were observed for these sons to have reduced sperm concentration, percent motile sperm and serum testosterone. A larger cohort is clearly needed to properly understand the effects of maternal obesity on male offspring fertility and sperm quality in humans. One potential consideration in human studies that examine associations between high BMI in men and their sperm parameters is that maternal BMI is generally not taken into account (and often these data are not available). The shared environment (e.g. social status, diet and lifestyle) and genetic similarity between mother and son will often mean that not only are the males being assessed obese, but they may have been exposed to increased maternal adiposity during gestation.

The effect of NMN administration on sperm has not been previously investigated. With energy being essential for sperm function, especially motility, these cells are highly reliant on the mitochondria. Obese men have been shown to have a reduction in sperm mitochondrial activity (Fariello et al. 2012). Therefore, as NMN supplementation can increase mitochondrial biogenesis and function, we hypothesised that sperm quality could be improved in obese mice. As expected (Uddin et al. 2016, 2017), in this experiment, NMN administration to mice fed a HFD reduced body weight, especially in the heaviest mice. However, we also found that NMN reduced sperm count and vitality and increased 8-OHdG.

One explanation for the increased oxidative stress (as indicated by $8-\mathrm{OHdG}$ ) was that the $\mathrm{NMN}$ administration was increasing ROS. We considered two mechanisms through which NMN could increase sperm ROS. Firstly, we and others have shown that NMN administration increases mitochondrial biogenesis and function (Gomes et al. 2013, Uddin et al. 2016, 2017). The mitochondria convert $\mathrm{NADH}$ to $\mathrm{NAD}^{+}$through a series of processes in energy metabolism and a by-product of this conversion 
is ROS, therefore, an increase in mitochondrial activity could increase ROS and oxidative stress. The NADHdependent oxidoreductase reaction in mitochondria is the main source of ROS in sperm so potentially perturbation of NADH levels by NMN administration could increase mitochondrial ROS output. In support of this possibility, measurement of sperm mtDNA copy number in the mice showed an interaction between maternal diet and NMN. MtDNA copy number was increased in offspring from a chow-fed mother, but decreased in offspring from a HFD-fed mother. However, it is unknown whether both of these effects could result in similar increases in $8-O H d G$. Secondly, ROS production from sperm plasma membranes is caused by NADPH oxidase (Shukla et al. 2005). Through a short biochemical pathway NMN could increase superoxide that is NMN to NADH (by NMNAT2), to NADPH (by NADH kinase) to superoxide (by NADPH oxidase).

Another possible mechanism that could explain the negative effects of NMN administration is that it could increase nicotinamide (NAM) levels as has been seen in mice with supplementation of another $\mathrm{NAD}^{+}$-precursor, nicotinamide riboside (NR) (Frederick et al. 2016). NAM is known to inhibit sirtuins (Bitterman et al. 2002) which are required for normal spermatogenesis (Coussens et al. 2008). However, this may contrast with a study that associated long-term supplementation with the sirtuinactivating drug resveratrol with increased mouse sperm DNA damage and 8-OHdG levels (Katen et al. 2016). It will be interesting to determine if other $\mathrm{NAD}^{+}$precursors such as nicotinic acid riboside (NAR), nicotinic acid mononucleotide (NaMN) and NR have negative effect on sperm quality at high levels.

To investigate whether NMN administration itself has negative impacts on sperm parameters, we examined a second cohort, independent of obesity in mice only fed regular chow. In this experiment, NMN was administered through drinking water at a slightly reduced dose (chronic rather than acute), which has nonetheless been shown to have beneficial effects on improving muscle blood vessel density and endurance in mice (Das et al. 2018). Unlike the maternal obesity experiment no differences in sperm count, viability or sperm mtDNA copy number were seen in these mice. Additionally, the FACS assays on live sperm showed no evidence for alterations to sperm oxidative stress or mitochondrial dysfunction. The only effect of NMN was a reduction in the HG proportion of viable sperm in the JC- 1 MMP assay. However, the ratio between $\mathrm{HO}$ and $\mathrm{HG}$ fluorescence was unaffected by NMN (Supplementary Fig. 2). In summary, minimal effects of NMN on sperm characteristics were observed under these conditions.

This oral administration cohort therefore suggests that chronic NMN administration can have beneficial effects on angiogenesis (Das et al. 2018) without impacting sperm quality. It may be that the effects of NMN on sperm quality, as assessed by $8-\mathrm{OHdG}$, seen in the maternal obesity cohort relate to the surge in NMN following acute injection versus gradual ingestion over the darkness phase (i.e. through the night), although further work would be required to test this. In particular, a future study investigating a group of mice receiving NMN through i.p. injection and another group receiving the same dose in drinking water would be enlightening.

Potential explanations for the differences in sperm states between the maternal obesity (i.p. injected NMN) cohort and the oral administration cohort may lie in the $\mathrm{NAD}^{+}$and NADH levels observed in the testes of representatives of each cohort (Fig. 4). These experiments showed large increases in $\mathrm{NAD}^{+}$and potentially $\mathrm{NADH}$ in the testes of the mice that had high sperm ROS, as well as low sperm count and vitality. These data support the possibility that increases in $\mathrm{NAD}^{+}$and/or $\mathrm{NADH}$ could alter ROS production through one, or several, of the mechanisms mentioned above, NADH-dependent oxidoreductase reactions, NADPH oxidases and Sirtuin-mediated reactions. These precise mechanistic explanations for how i.p. NMN administration could cause sperm oxidative stress could in future be investigated through mass-spectrometry of testes or sperm for metabolites such as NAM, NADPH and NADH. Additionally, live sperm JC-1 staining in sperm from mice that have had i.p. NMN administration could reveal if the sperm deficits (vitality and 8-OHdG) are associated with altered mitochondrial activity.

\section{Conclusions}

Our data suggest that both maternal obesity and postweaning diet-induced obesity are risk factors for reduced sperm quality. However, ways in which these two exposures impact sperm may differ and are not additive. Our examination of the effects of NMN supplementation point to dose- and/or route-of-administration differences in the consequences for sperm quality, which invites further investigation.

\section{Supplementary data}

This is linked to the online version of the paper at https://doi.org/10.1530/REP-18-0574.

\section{Declaration of interest}

D A S is a founder, equity owner, board member, advisor to, director of, consultant to, investor in and/or inventor on patents licensed to Vium, Jupiter Orphan Therapeutics, Cohbar, Galilei Biosciences, GlaxoSmithKline, OvaScience, EMD Millipore, Wellomics, Inside Tracker, Caudalie, Bayer Crop Science, Longwood Fund, Zymo Research, EdenRoc Sciences (and affiliates Arc-Bio, Dovetail Genomics, Claret Bioscience, Revere Biosensors, UpRNA and MetroBiotech (an NAD booster company), Liberty Biosecurity), Life Biosciences (and affiliates Selphagy, Senolytic Therapeutics, Spotlight 
Biosciences, Animal Biosciences, Iduna, Immetas, Prana, Continuum Biosciences, Jumpstart Fertility (an NAD booster company), and Lua Communications). D A S sits on the board of directors of both companies. D A S is an inventor on a patent application filed by Mayo Clinic and Harvard Medical School that has been licensed to Elysium Health; his personal royalty share is directed to the Sinclair lab. For more information see https://genetics.med.harvard.edu/sinclair-test/people/sinclairother.php. Prof. R John Aitken is on the editorial board of Reproduction. Prof. Aitken was not involved in the review or editorial process for this paper, on which he is listed as an author. The other authors have nothing to disclose.

\section{Funding}

This research was funded by National Health and Medical Research Council (NHMRC) project grant \#1044295 to M J M and D A S. G M U was supported by a University International Postgraduate Award (UIPA), UNSW Sydney. DAS was supported by The Glenn Foundation for Medical Research, and NIH R01AG028730 / R01DK100263

\section{Author contribution statement}

M J M, D A S, N A Y, R J A and A D contributed to experimental conception and design. G M U, N A Y, C M, A D, C M, J Y, H S C and S W performed the experiments. N A Y, G M U, C M and $M J M$ analysed the data. N A Y, C M and M J M wrote the first draft of the paper. All authors reviewed and approved the final manuscript.

\section{Acknowledgements}

The authors wish to thank to Chris Brownlee and Emma Johansson Beves of the Biological Resources Imaging Laboratory at UNSW for their guidance in flow cytometry.

\section{References}

Agarwal A, Virk G, Ong C \& du Plessis SS 2014 Effect of oxidative stress on male reproduction. World Journal of Men's Health 32 1-17. (https://doi. org/10.5534/wjmh.2014.32.1.1)

Ahmed-Farid OAH, Nasr M, Ahmed RF \& Bakeer RM 2017 Beneficial effects of curcumin nanoemulsion on spermatogenesis and reproductive performance in male rats under protein deficient diet model: enhancement of sperm motility, conservancy of testicular tissue integrity, cell energy and seminal plasma amino acids content. Journal of Biomedical Science 24 66. (https://doi.org/10.1186/s12929-017-0373-5)

Ambrosetti V, Guerra M, Ramirez LA, Reyes A, Alvarez D, Olguin S, Gonzalez-Manan D, Fernandois D, Sotomayor-Zarate R \& Cruz G 2016 Increase in endogenous estradiol in the progeny of obese rats is associated with precocious puberty and altered follicular development in adulthood. Endocrine 53 258-270. (https://doi.org/10.1007/s12020016-0858-0)

Avci ME, Sanlikan F, Celik M, Avci A, Kocaer M \& Gocmen A 2015 Effects of maternal obesity on antenatal, perinatal and neonatal outcomes. Journal of Maternal-Fetal and Neonatal Medicine 28 2080-2083. (https:// doi.org/10.3109/14767058.2014.978279)

Bahari H, Caruso V \& Morris MJ 2013 Late-onset exercise in female rat offspring ameliorates the detrimental metabolic impact of maternal obesity. Endocrinology $154 \quad 3610-3621 . \quad$ (https://doi.org/10.1210/ en.2013-1059)
Bandel I, Bungum M, Richtoff J, Malm J, Axelsson J, Pedersen HS, Ludwicki JK, Czaja K, Hernik A, Toft G et al. 2015 No association between body mass index and sperm DNA integrity. Human Reproduction 30 1704-1713. (https://doi.org/10.1093/humrep/dev111)

Baur JA, Pearson KJ, Price NL, Jamieson HA, Lerin C, Kalra A, Prabhu VV, Allard JS, Lopez-Lluch G, Lewis K et al. 2006 Resveratrol improves health and survival of mice on a high-calorie diet. Nature 444 337-342. (https://doi.org/10.1038/nature05354)

Bertoldo MJ, Uddin GM, Youngson NA, Agapiou D, Walters KA, Sinclair DA, Morris MJ \& Gilchrist RB 2018 Multigenerational obesityinduced perturbations in oocyte-secreted factor signalling can be ameliorated by exercise and nicotinamide mononucleotide. Human Reproduction Open 2018 hoy010. (https://doi.org/10.1093/hropen/ hoy010)

Binet MT, Doyle CJ, Williamson JE \& Schlegel P 2014 Use of JC-1 to assess mitochondrial membrane potential in sea urchin sperm. Journal of Experimental Marine Biology and Ecology 452 91-100. (https://doi. org/10.1016/j.jembe.2013.12.008)

Bitterman KJ, Anderson RM, Cohen HY, Latorre-Esteves M \& Sinclair DA 2002 Inhibition of silencing and accelerated aging by nicotinamide, a putative negative regulator of yeast sir2 and human SIRT1. Journal of Biological Chemistry 277 45099-45107. (https://doi.org/10.1074/jbc. M205670200)

Boney CM, Verma A, Tucker R \& Vohr BR 2005 Metabolic syndrome in childhood: association with birth weight, maternal obesity, and gestational diabetes mellitus. Pediatrics 115 e290-e296. (https://doi. org/10.1542/peds.2004-1808)

Callaway LK, Prins JB, Chang AM \& McIntyre HD 2006 The prevalence and impact of overweight and obesity in an Australian obstetric population. Medical Journal of Australia 184 56-59. (https://doi. org/10.5694/j.1326-5377.2006.tb00115.x)

Cardozo E, Pavone ME \& Hirshfeld-Cytron JE 2011 Metabolic syndrome and oocyte quality. Trends in Endocrinology and Metabolism 22 103-109. (https://doi.org/10.1016/j.tem.2010.12.002)

Chavarro JE, Toth TL, Wright DL, Meeker JD \& Hauser R 2010 Body mass index in relation to semen quality, sperm DNA integrity, and serum reproductive hormone levels among men attending an infertility clinic. Fertility and Sterility 93 2222-2231. (https://doi.org/10.1016/j. fertnstert.2009.01.100)

Christante CM, Taboga SR, Pinto-Fochi ME \& Goes RM 2013 Maternal obesity disturbs the postnatal development of gonocytes in the rat without impairment of testis structure at prepubertal age. Reproduction 146 549-558. (https://doi.org/10.1530/REP-13-0037)

Considine RV, Sinha MK, Heiman ML, Kriauciunas A, Stephens TW, Nyce MR, Ohannesian JP, Marco CC, McKee LJ \& Bauer TL 1996 Serum immunoreactive-leptin concentrations in normal-weight and obese humans. New England Journal of Medicine 334 292-295. (https://doi. org/10.1056/NEJM199602013340503)

Coussens M, Maresh JG, Yanagimachi R, Maeda G \& Allsopp R 2008 Sirt1 deficiency attenuates spermatogenesis and germ cell function. PLoS ONE 3 e1571. (https://doi.org/10.1371/journal.pone.0001571)

Craig JR, Jenkins TG, Carrell DT \& Hotaling JM 2017 Obesity, male infertility, and the sperm epigenome. Fertility and Sterility 107 848-859. (https://doi.org/10.1016/j.fertnstert.2017.02.115)

Das A, Huang GX, Bonkowski MS, Longchamp A, Li C, Schultz MB, Kim LJ, Osborne B, Joshi S, Lu Y et al. 2018 Impairment of an endothelial $\mathrm{NAD}(+)-\mathrm{H} 2 \mathrm{~S}$ signaling network is a reversible cause of vascular aging. Cell 173 74.e20-89.e20. (https://doi.org/10.1016/j.cell.2018.02.008)

Dupont C, Faure C, Sermondade N, Boubaya M, Eustache F, Clement P, Briot P, Berthaut I, Levy V, Cedrin-Durnerin I et al. 2013 Obesity leads to higher risk of sperm DNA damage in infertile patients. Asian Journal of Andrology 15 622-625. (https://doi.org/10.1038/aja.2013.65)

Elshenawy S \& Simmons R 2016 Maternal obesity and prenatal programming. Molecular and Cellular Endocrinology 435 2-6. (https:// doi.org/10.1016/j.mce.2016.07.002)

Erdemir F, Atilgan D, Markoc F, Boztepe O, Suha-Parlaktas B \& Sahin S 2012 The effect of diet induced obesity on testicular tissue and serum oxidative stress parameters. Actas Urologicas Espanolas 36 153-159. (https://doi.org/10.1016/j.acuro.2011.06.019)

Evenson D \& Jost L 2000 Sperm chromatin structure assay is useful for fertility assessment. Methods in Cell Science 22 169-189. (https://doi. org/10.1023/A:1009844109023) 
Fan Y, Liu Y, Xue K, Gu G, Fan W, Xu Y \& Ding Z 2015 Diet-induced obesity in male $\mathrm{C} 57 \mathrm{BL} / 6$ mice decreases fertility as a consequence of disrupted blood-testis barrier. PLOS ONE 10 e0120775. (https://doi. org/10.1371/journal.pone.0120775)

Fariello RM, Pariz JR, Spaine DM, Cedenho AP, Bertolla RP \& Fraietta R 2012 Association between obesity and alteration of sperm DNA integrity and mitochondrial activity. BJU International 110 863-867. (https://doi. org/10.1111/j.1464-410X.2011.10813.x)

Fernandez JL, Muriel L, Rivero MT, Goyanes V, Vazquez R \& Alvarez JG 2003 The sperm chromatin dispersion test: a simple method for the determination of sperm DNA fragmentation. Journal of Andrology 24 59-66. (https://doi.org/10.1002/j.1939-4640.2003.tb02641.x)

Frederick DW, Loro E, Liu L, Davila A, Chellappa K, Silverman IM, Quinn WJ, Gosai SJ, Tichy ED, Davis JG et al. 2016 Loss of NAD homeostasis leads to progressive and reversible degeneration of skeletal muscle. Cell Metabolism 24 269-282. (https://doi.org/10.1016/j. cmet.2016.07.005)

Gariani K, Menzies KJ, Ryu D, Wegner CJ, Wang X, Ropelle ER, Moullan N, Zhang H, Perino A, Lemos V et al. 2016 Eliciting the mitochondrial unfolded protein response by nicotinamide adenine dinucleotide repletion reverses fatty liver disease in mice. Hepatology 63 1190-1204. (https://doi.org/10.1002/hep.28245)

Gavriliouk D \& Aitken RJ 2015 Damage to sperm DNA mediated by reactive oxygen species: its impact on human reproduction and the health trajectory of offspring. Advances in Experimental Medicine and Biology 868 23-47. (https://doi.org/10.1007/978-3-319-18881-2_2)

Ge ZJ, Liang QX, Hou Y, Han ZM, Schatten H, Sun QY \& Zhang CL 2014 Maternal obesity and diabetes may cause DNA methylation alteration in the spermatozoa of offspring in mice. Reproductive Biology and Endocrinology 12 29. (https://doi.org/10.1186/1477-7827-12-29)

Gomes AP, Price NL, Ling AJY, Moslehi JJ, Montgomery MK, Rajman L, White JP, Teodor JS, Wrann CD, Hubbard BP et al. 2013 Declining $\mathrm{NAD}(+)$ induces a pseudohypoxic state disrupting nuclear-mitochondrial communication during aging. Cell 155 1624-1638. (https://doi. org/10.1016/j.cell.2013.11.037)

Hjollund NH, Bonde JP, Jensen TK \& Olsen J 2000 Diurnal scrotal skin temperature and semen quality. The Danish first pregnancy planner study team. International Journal of Andrology 23 309-318. (https://doi. org/10.1046/j.1365-2605.2000.00245.x)

Houston BJ, Nixon B, Martin JH, De Iuliis GN, Trigg NA, Bromfield EG, McEwan KE \& Aitken RJ 2018 Heat exposure induces oxidative stress and DNA damage in the male germ line. Biology of Reproduction 98 593-606. (https://doi.org/10.1093/biolre/ioy009)

Huang L, Haratake K, Miyahara H \& Chiba T 2016 Proteasome activators, PA28 gamma and PA200, play indispensable roles in male fertility. Scientific Reports 6 23171. (https://doi.org/10.1111/and.13224)

Jensen TK, Andersson AM, Jorgensen N, Andersen AG, Carlsen E, Petersen JH \& Skakkebaek NE 2004 Body mass index in relation to semen quality and reproductive hormones among 1,558 Danish men. Fertility and Sterility 82 863-870. (https://doi.org/10.1016/j. fertnstert.2004.03.056)

Katen AL, Stanger SJ, Anderson AL, Nixon B \& Roman SD 2016 Chronic acrylamide exposure in male mice induces DNA damage to spermatozoa; potential for amelioration by resveratrol. Reproductive Toxicology 63 1-12. (https://doi.org/10.1016/j.reprotox.2016.05.004)

Kocer A, Henry-Berger J, Noblanc A, Champroux A, Pogorelcnik R, Guiton R, Janny L, Pons-Rejraji H, Saez F, Johnson GD et al. 2015 Oxidative DNA damage in mouse sperm chromosomes: size matters. Free Radical Biology and Medicine 89 993-1002. (https://doi.org/10.1016/j. freeradbiomed.2015.10.419)

Kort HI, Massey JB, Elsner CW, Mitchell-Leef D, Shapiro DB, Witt MA \& Roudebush WE 2006 Impact of body mass index values on sperm quantity and quality. Journal of Andrology 27 450-452. (https://doi. org/10.2164/jandrol.05124)

Liu Y \& Ding Z 2017 Obesity, a serious etiologic factor for male subfertility in modern society. Reproduction $\mathbf{1 5 4}$ R123-R131. (https://doi. org/10.1530/REP-17-0161)

Malik AN \& Czajka A 2013 Is mitochondrial DNA content a potential biomarker of mitochondrial dysfunction? Mitochondrion 13 481-492. (https://doi.org/10.1016/j.mito.2012.10.011)

Martin KE, Grivell RM, Yelland LN \& Dodd JM 2015 The influence of maternal BMI and gestational diabetes on pregnancy outcome. Diabetes
Research and Clinical Practice 108 508-513. (https://doi.org/10.1016/j. diabres.2014.12.015)

Moustafa MH, Sharma RK, Thornton J, Mascha E, Abdel-Hafez MA, Thomas AJ \& Agarwal A 2004 Relationship between ROS production, apoptosis and DNA denaturation in spermatozoa from patients examined for infertility. Human Reproduction 19 129-138. (https://doi. org/10.1093/humrep/deh024)

Oben JA, Mouralidarane A, Samuelsson AM, Matthews PJ, Morgan ML, Mckee C, Soeda J, Fernandez-Twinn DS, Martin-Gronert MS, Ozanne SE et al. 2010 Maternal obesity during pregnancy and lactation programs the development of offspring non-alcoholic fatty liver disease in mice. Journal of Hepatology 52 913-920. (https://doi.org/10.1016/j. jhep.2009.12.042)

Oliveira JBA, Petersen CG, Mauri AL, Vagnini LD, Renzi A, Petersen B, Mattila M, Dieamant F, Baruffi RLR \& Franco JG 2018 Association between body mass index and sperm quality and sperm DNA integrity. A large population study. Andrologia 50 e12889. (https://doi.org/10.1111/ and.12889)

Ramlau-Hansen CH, Nohr EA, Thulstrup AM, Bonde JP, Storgaard L \& Olsen J 2007 Is maternal obesity related to semen quality in the male offspring? A pilot study. Human Reproduction 22 2758-2762. (https:// doi.org/10.1093/humrep/dem219)

Reynolds RM, Allan KM, Raja EA, Bhattacharya S, McNeill G, Hannaford PC, Sarwar N, Lee AJ, Bhattacharya S \& Norman JE 2013 Maternal obesity during pregnancy and premature mortality from cardiovascular event in adult offspring: follow-up of 1323275 person years. BMJ-British Medical Journal 347 f4539. (https://doi.org/10.1136/bmj.f4539)

Rodriguez-Gonzalez GL, Vega CC, Boeck L, Vazquez M, Bautista CJ, ReyesCastro LA, Saldana O, Lovera D, Nathanielsz PW \& Zambrano E 2015 Maternal obesity and overnutrition increase oxidative stress in male rat offspring reproductive system and decrease fertility. International Journal of Obesity 39 549-556. (https://doi.org/10.1038/ijo.2014.209)

Santos M, Rodriguez-Gonzalez GL, Ibanez C, Vega CC, Nathanielsz PW \& Zambrano E 2015 Adult exercise effects on oxidative stress and reproductive programming in male offspring of obese rats. American Journal of Physiology: Regulatory, Integrative and Comparative Physiology 308 R219-R225. (https://doi.org/10.1152/ajpregu.00398.2014)

Shukla S, Jha RK, Laloraya M \& Kumar PG 2005 Identification of nonmitochondrial NADPH oxidase and the spatio-temporal organization of its components in mouse spermatozoa. Biochemical and Biophysical Research Communications 331 476-483. (https://doi.org/10.1016/j. bbrc.2005.03.198)

Smith TB, Baker MA, Connaughton HS, Habenicht U \& Aitken RJ 2013 Functional deletion of Txndc2 and Txndc3 increases the susceptibility of spermatozoa to age-related oxidative stress. Free Radical Biology and Medicine 65 872-881. (https://doi.org/10.1016/j. freeradbiomed.2013.05.021)

Soubry A, Murphy SK, Wang F, Huang Z, Vidal AC, Fuemmeler BF, Kurtzberg J, Murtha A, Jirtle RL, Schildkraut JM et al. 2015 Newborns of obese parents have altered DNA methylation patterns at imprinted genes. International Journal of Obesity 39 650-657. (https://doi. org/10.1038/ijo.2013.193)

Starling AP, Brinton JT, Glueck DH, Shapiro AL, Harrod CS, Lynch AM, Siega-Riz AM \& Dabelea D 2015 Associations of maternal BMI and gestational weight gain with neonatal adiposity in the Healthy Start study. American Journal of Clinical Nutrition 101 302-309. (https://doi. org/10.3945/ajcn.114.094946)

Sullivan EA, Dickinson JE, Vaughan GA, Peek MJ, Ellwood D, Homer CSE, Knight M, McLintock C, Wang A, Pollock W et al. 2015 Maternal superobesity and perinatal outcomes in Australia: a national populationbased cohort study. BMC Pregnancy and Childbirth 15 322. (https://doi. org/10.1186/s12884-015-0693-y)

Tsoulis MW, Chang PE, Moore CJ, Chan KA, Gohir W, Petrik JJ, Vickers MH, Connor KL \& Sloboda DM 2016 Maternal high-fat diet-induced loss of fetal oocytes is associated with compromised follicle growth in adult rat offspring. Biology of Reproduction 94 94. (https://doi.org/10.1095/ biolreprod.115.135004)

Uddin GM, Youngson NA, Sinclair DA \& Morris MJ $2016 \mathrm{Head}$ to head comparison of short-term treatment with the $\mathrm{NAD}(+)$ precursor nicotinamide mononucleotide (NMN) and 6 weeks of exercise in obese female mice. Frontiers in Pharmacology 7 258. (https://doi.org/10.3389/ fphar.2016.00258) 
Uddin GM, Youngson NA, Doyle BM, Sinclair DA \& Morris MJ 2017 Nicotinamide mononucleotide (NMN) supplementation ameliorates the impact of maternal obesity in mice: comparison with exercise. Scientific Reports 7 15063. (https://doi.org/10.1038/s41598-017-14866-z)

Vorilhon S, Brugnon F, Kocer A, Dollet S, Bourgne C, Berger M, Janny L, Pereira B, Aitken RJ, Moazamian A et al. 2018 Accuracy of human sperm DNA oxidation quantification and threshold determination using an 8-OHdG immuno-detection assay. Human Reproduction 33 553-562. (https://doi.org/10.1093/humrep/dey038)

Wai T, Ao A, Zhang X, Cyr D, Dufort D \& Shoubridge EA 2010 The role of mitochondrial DNA copy number in mammalian fertility. Biology of Reproduction 83 52-62. (https://doi.org/10.1095/ biolreprod.109.080887)

World Health Organisation 2015 Obesity and overweight (Fact sheet No. 311). (available at: http://www.who.int/mediacentre/factsheets/fs311/ en./)

Yang Y \& Sauve AA 2016 NAD(+) metabolism: bioenergetics, signaling and manipulation for therapy. Biochimica et Biophysica Acta 1864 1787-1800. (https://doi.org/10.1016/j.bbapap.2016.06.014)
Yeung CH, Anapolski M, Sipila P, Wagenfeld A, Poutanen M, Huhtaniemi I, Nieschlag E \& Cooper TG 2002 Sperm volume regulation: maturational changes in fertile and infertile transgenic mice and association with kinematics and tail angulation. Biology of Reproduction 67 269-275. (https://doi.org/10.1095/biolreprod67.1.269)

Yoshino J, Mills KF, Yoon MJ \& Imai SI 2011 Nicotinamide mononucleotide, a key $\mathrm{NAD}(+)$ intermediate, treats the pathophysiology of diet- and ageinduced diabetes in mice. Cell Metabolism 14 528-536. (https://doi. org/10.1016/j.cmet.2011.08.014)

Received 4 November 2018

First decision 20 December 2018

Revised manuscript received 2 May 2019

Accepted 30 May 2019 